Accelerator Division

Alternating Gradient Synchrotron Department

BROOKHAVEN NATIONAL LABORATORY

Upton, New York 11973

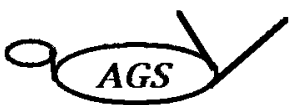

Accelerator Division

Technical Note

AGS/AD/Tech Note No. 458

\title{
FEB/V(p):The FY1996 Commissioning with Proton Beam and Run for
}

the $V$-Line and V-Target Commissioning

\author{
M. (Sanki) Tanaka and L. Ahrens \\ (sanki@bnl.gov and ahrens@bnldag.ags.bnl.gov)) \\ 10 March 1997
}

Version 1.1 : changed TN No. 459 to 458.

\begin{abstract}
On 3 May 1996 the FEB system was successfully commissioned with low intensity proton beam $\left(\sim 0.5 \cdot 10^{12} \mathrm{ppb}\right)$, by accelerating and extracting just one bunch per AGS cycle at $24.0 \mathrm{GeV} / \mathrm{c}$ to the V-dump. The FEB/SBE(p) run continued parasitically with the HEP/SEB program using the context switching on-request mode for the $\mathrm{V}$ line, $\mathrm{V}$ target and secondary V2 line

commissioning until 3 July 1996 . The bunch intensity was gradually increased up to $\sim 5 \times 10^{12} \mathrm{ppb}$ for the final dedicated high intensity $\mathrm{V}$ target test that lasted for 30 minutes. However, there were substantial beam losses at the H10 septum magnet and its downstream ssH11-H13 partially due to a larger transverse beam emittance, a wider momentum spread and a longer bunch length for a high intensity machine setup. A test of double single-bunch extraction (2 SBEs) per AGS cycle was carried out by bypassing the resonant charging module of the H10 septum power supply that was not quite ready for operation. All FEB devices double-pulsed at $100 \mathrm{~ms}$ apart at equal strength and the first SBE was successfully performed but not the second one. It was found that the radial loop system could not keep the second bunch left in the ring at the same mean radius and the beam drifted away outward, crashing to the G10 kicker and the H10 septum.
\end{abstract}




\section{INTRODUCTION}

For the BNL muon g-2 experiment (E825), which has constructed a $14 \mathrm{~m}$ diameter superferric muon storage ring with $\mathrm{B}=1.5 \mathrm{~T}$ in order to improve the previous CERN measurement of the anomalous muon magnetic moment ( $\left.a_{\mu}\right)$ by a factor of 20 , the FEB extraction system must meet the following requirements: (1) extract the bunched proton beam at $20-24 \mathrm{GeV} / \mathrm{c}$ and up to full intensity to the newly constructed $\mathrm{V}$ target through the $\mathrm{U}$ line for $3.1 \mathrm{GeV} / \mathrm{c} \pi / \mu$ production, and (2) perform multiple single-bunch extraction at $30 \mathrm{~Hz}$ up to 8 times per AGS cycle [1].

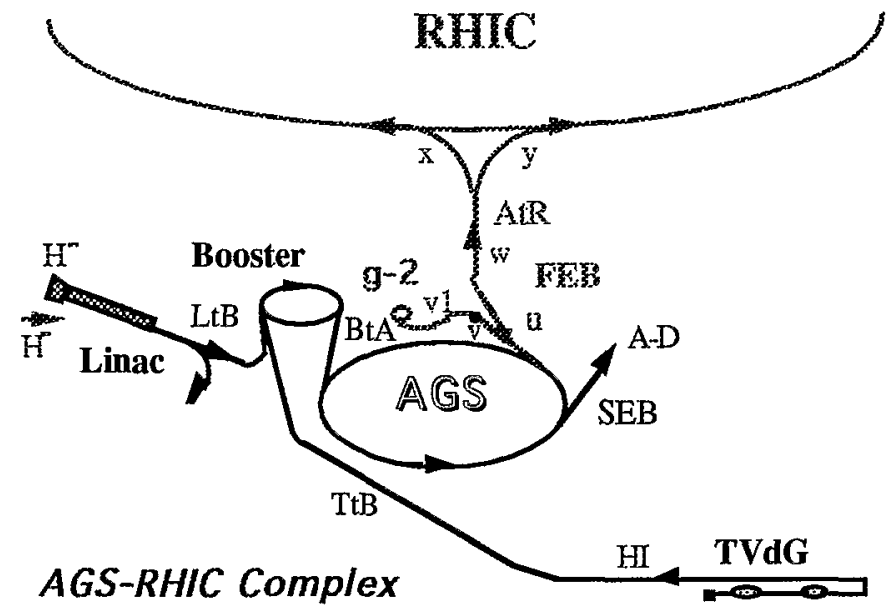

Figure 1: Schematic view of the AGS-RHIC complex.

In the May-July of 1996, both the FEB system and the V line, V target and V1 line were commissioned with proton beam. The previous FY96 FEB/AtR(Au) commissioning and run is described in ref.[2]. The schematic layout of the AGS Accelerator Complex with the g-2 storage ring and RHIC is shown in Figure 1.

\section{THE FEB EXTRACTION SYSTEM}

\section{II.A. Machine and Beam Parameters}

The new system is described in ref.[4] and was designed based on the 1991 FEB conceptual design report [3] which had assumed that

1. the operational momentum range: $22 \leq \mathrm{p} \leq 29 \mathrm{GeV} / \mathrm{c} / \mathrm{charge}$,

2. the maximum $95 \%$ normalized transverse emittance of the high intensity proton beam:

$$
\varepsilon_{\mathrm{h}, \mathrm{v}}^{*}(95 \%)=6 \cdot \sigma^{2} / \beta \cdot(\mathrm{p} / \mathrm{m})=\sim 50 \pi \mathrm{mm}-\mathrm{mrad},
$$

where $\sigma$ is the standard deviation of the beam size due to the transverse emittance,

3. the maximum total momentum spread: $(\mathrm{dp} / \mathrm{p})$ full $= \pm 0.2 \%$,

4. the maximum full bunch length: $l_{\text {bunch }}=\sim 50 \mathrm{~ns}$,

5. the AGS rf harmonic number: $\mathrm{h}=12$.

In 1994 the AGS rf harmonic number was changed from $h=12$ to 8 in AGS (from 3 to 2 in Booster) for high intensity proton operation in order to accelerate the high intensity beam without difficulties along with increasing the bunch length, momentum spread and transverse emittance. It should be noted that for the SEB users, their main interest is uniformity of the beam spill and beam intensity. The actual measured values of $\varepsilon^{*}$, $\mathrm{dp} / \mathrm{p}$ and $\mathrm{l}_{\mathrm{b}}$ for the AGS beam strongly depend on the machine condition, especially the exact high intensity setup. Though the high intensity values for the FEB(p) operation have not yet been optimized, it is now apparent that the values of $\varepsilon^{*}, \mathrm{dp} / \mathrm{p}$ and lbunch are larger than the FEB design values and may cause some problems (i.e., beam loss). Due to its high intensity operation for the g- 2 experiment, it is critical that the FEB 
system can practically achieve $\sim 100 \%$ extraction efficiency. The AGS basic machine parameters and performance of the FY1995-96 proton SEB operation are summarized in Table 1.

Table 1: AGS machine and recent SEB proton beam parameters.

\begin{tabular}{|l|ll|}
\hline Circumference & $\mathrm{C}=807.091$ & {$[\mathrm{~m}]$} \\
Curvature & $\rho=85.37$ & {$[\mathrm{~m}]$} \\
Revolution time & $\mathrm{t}_{\mathrm{rev}}=2.692$ & {$[\mu \mathrm{s}]$} \\
Tune & $\mathrm{Q}_{\mathrm{h}} \approx \mathrm{Q}_{\mathrm{v}}=\sim 8.7$ & \\
Beta functions & $\beta_{\mathrm{h}, \mathrm{v}}=22.5-10.5$ & {$[\mathrm{~m}]$} \\
Dispersion Fun. & $\mathrm{D}_{\mathrm{x}} \mathrm{max}=2.20$ & {$[\mathrm{~m}]$} \\
No. of bunches & $\mathrm{N}_{\mathrm{b}}=8(2 \mathrm{x} 4)^{\dagger}$ & before debunching \\
Gap bet. bunches & 336 & {$[\mathrm{~ns}]$} \\
Typical intensity & $5.5 \cdot 10^{13}$ & {$[\mathrm{ppp}]$} \\
Typical cycle & 3.2 & {$[\mathrm{~s}]$} \\
Typical spill length & 1.2 & {$[\mathrm{~s}]$} \\
Momentum & $\mathrm{p}^{*}=25.5$ & {$[\mathrm{GeV} / \mathrm{c}]$} \\
Trans. emittance & $\varepsilon^{*} \mathrm{~T}(95 \%)=\sim 80 \pi ?$ & {$[\mathrm{~mm}-\mathrm{mrad}]$} \\
Long. emittance & $\varepsilon_{\mathrm{L}}(95 \%)=\sim 5 ?$ & {$[\mathrm{eV}-\mathrm{s} / \mathrm{bunch}]$} \\
Full bunch length & $\mathrm{l}_{\mathrm{b}=100-120}$ & {$[\mathrm{~ns}]$ bf debunching } \\
\hline
\end{tabular}

$\uparrow 4$ Booster transfers, each has 2 bunches.

The following table lists the present or expected beam parameters and performance of the AGS Complex for FEB operation for various users.

Table 2: FEB beam parameters.

\begin{tabular}{|c|c|c|c|c|}
\hline 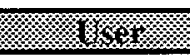 & \multicolumn{4}{|c|}{ 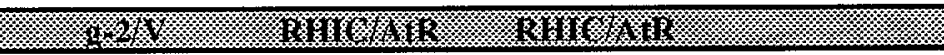 } \\
\hline Particle & Protons & Protons & Heavy ions & \\
\hline Momentum & $24^{\dagger}$ & $\begin{array}{l}28.0 \\
(25.5 \text { for pol. } p)\end{array}$ & $\begin{array}{l}28.0 \\
28.0 \times(79 / 197)\end{array}$ & $\begin{array}{l}x[\mathrm{Z} / \mathrm{A}] \mathrm{GeV} / \mathrm{c} / \mathrm{N} \\
\text { for }\left(\mathrm{Au}^{77+}\right)\end{array}$ \\
\hline $\mathrm{N}_{\mathrm{SBE}} /$ cycle & $1,2,,, 8(12)^{\dagger \dagger}$ & $4 \times(15 \times 2)$ & $4 \times(15 \times 2)$ for & two rings \\
\hline $\mathrm{N}_{\text {ion/bunch }}$ & $7500(5000)^{\dagger \dagger}$ & 100 & $1(\mathrm{Au}), 6(\mathrm{Si})$ & {$\left[10^{9}\right]$} \\
\hline$\varepsilon_{\mathrm{h}, \mathrm{V}}^{*}(95 \%)$ & $\sim 80 \pi ?(50 \pi)^{\dagger \dagger}$ & $20 \pi$ & $10 \pi$ & [mm-mrad] \\
\hline$\varepsilon_{\mathrm{L}}(95 \%)$ & $2.5 ?$ & 0.3 & 0.3 & [eV-s/bunch] \\
\hline lbunch(full) & $\sim 100 ?(50)^{\dagger \dagger}$ & 12 & 17 & [ns] \\
\hline$(\mathrm{dp} / \mathrm{p})_{\text {full }}$ & $\pm 0.2 ?$ & \pm 0.06 & \pm 0.10 & [\%] \\
\hline
\end{tabular}

$\dagger$ The optimum momentum might be $20 \mathrm{GeV} / \mathrm{c}$ for $3.1 \mathrm{GeV} / \mathrm{c} \pi / \mu$ production.

$\dagger$ Values in () were the original design assumptions.

\section{II.B. Extraction Scheme}

The FEB system consists of a fast multi-pulsing kicker at straight section G10 [FKG10] followed by a thick septum ejector magnet at ssH10 [SMH10]. In Figure 2, we show a schematic layout of the FEB extraction components and the extraction orbit bumps. To minimize the required voltage on pulsing the fast kicker, the kicker is a C-type open ferrite magnet with a pole tip. The center of the kicker aperture is placed about $64 \mathrm{~mm}$ from the central orbit at the middle of ssG10. A few msec before each single-bunch extraction two extraction orbit bumps [BLWG09/H11] and SMH10 are excited to bring the beam into the aperture of the kicker and adjacent to the septum of the ejector. During extraction, the kicker is synchronized and phased to the bunches and triggered every specified time $(\leq 30 \mathrm{~Hz})$ to send one bunch at a time into the ejector, which gives an additional larger kick to extract the bunch out of the ring. All FEB devices are powered by capacitordischarge power supplies giving an approximately half-sine excitation and are so-called CLD (Complex Logic Device) and multi-pulsing devices. 


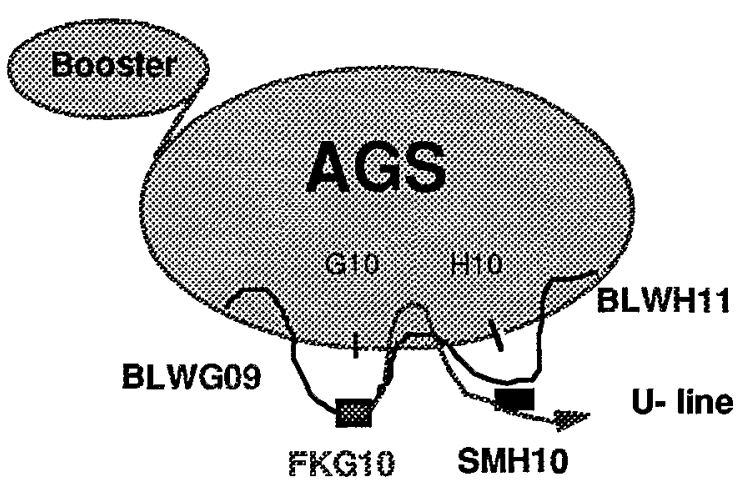

Figure 2: Layout of the AGS FEB components.

The FEB orbit bumps are slowest and are discharged about $3.2 \mathrm{~ms}$ before extraction time. The SMH10 septum is discharged about $1.3 \mathrm{~ms}$ before extraction. The FKG10 kicker with $380 \mathrm{~ns}$ base-width is trigger by the rf synchronized signal just before extraction and has a fine time-delay module in a nsec interval. The basic parameters of the kicker, ejector and extraction bumps are summarized in Table 3.

Table 3: FEB magnet parameters.

\begin{tabular}{|c|c|c|c|}
\hline : & Miske- & Yistor & Gunus \\
\hline Name & FKG10 & SMH10 & BLWG09/H11 \\
\hline Location & ssG10 & ssH10 & $\begin{array}{l}\text { F8/9-G2/3, G16/17 } \\
\mathrm{H} 4 / 5, \mathrm{H} 18 / 19-\mathrm{I} 12 / 13\end{array}$ \\
\hline$\theta_{\max }[\mathrm{mrad}]$ & -2.0 & 22.0 & 2(4)/pair \\
\hline Waveform & half-sine & half-sine & half-sine \\
\hline Septum [mm] & - - & 10 & $-\cdots$ \\
\hline Aperture $[\mathrm{mm}]$ & $32(\mathrm{~h}) \times 22(\mathrm{v})$ & $72 \times 25$ & full \\
\hline $\mathrm{B}_{\max }[\mathrm{T}]$ & 0.1 & 1.0 & $0.05(0.1)$ \\
\hline Length[m] & 2.4 & 2.1 & -- \\
\hline tbase-width & $380 \mathrm{~ns}$ & $2.6 \mathrm{~ms}$ & $7 \mathrm{~ms}$ \\
\hline$I_{\max }[\mathrm{kA}]$ & 2.0 & 23 & $0.6(1.2)$ \\
\hline Tolerance $[\%]$ & $< \pm 0.9$ & $< \pm 0.09$ & $< \pm 0.8$ \\
\hline
\end{tabular}

$\dagger$ Its power supply does not yet have an operational resonant charging module that allows SMH10 to pulse at $30 \mathrm{~Hz}$ rather $10 \mathrm{~Hz}$.

t† Without trim backleg windings.

The displacement, $\Delta \mathrm{x}_{\mathrm{S}}$, and angle change, $\Delta \mathrm{x}_{\mathrm{S}}$, between the circulating bunches and kicked bunch at SMH10us are proportional to the kick strength $\left(\theta_{k}\right)$ for the bunch entering the aperture of FKG10:

$$
\begin{aligned}
& \Delta \mathrm{x}_{\mathrm{S}}[\mathrm{mm}]=\theta_{\mathrm{k}} \cdot \sqrt{\beta_{\mathrm{k}}} \cdot \sqrt{\mathrm{B}_{\mathrm{S}}} \cdot \sin (\Delta \mu)=-17.15 \cdot \theta_{\mathrm{k}}[\mathrm{mrad}] \cdot \\
& \Delta \mathrm{x}_{\mathrm{S}}[\mathrm{mrad}]=\theta_{\mathrm{k}} \cdot\left(\sqrt{\mathfrak{B}_{\mathrm{K}}} / \sqrt{\mathrm{B}_{\mathrm{S}}}\right)\left(\cos (\Delta \mu)-\alpha_{\mathrm{S}} \cdot \sin (\Delta \mu)\right)=1.083 \cdot \theta_{\mathrm{k}}[\mathrm{mrad}]
\end{aligned}
$$

where $\Delta \mu=0.683 \cdot 2 \pi$ is the betatron phase advance from FKG10ds to SMH10us, $B$ and $\alpha$ are lattice parameters. Using $\varepsilon^{*} \mathrm{~T}(95 \%)=80 \pi \mathrm{mm}-\mathrm{mrad},(\Delta \mathrm{p} / \mathrm{p})_{\mathrm{rms}}=0.001, \mathrm{p}=24 \mathrm{GeV} / \mathrm{c}, 10 \mathrm{~mm}$ septum thickness and $\sim 2 \mathrm{~mm}$ safety margin at both sides of the septum, we have $\Delta \mathrm{x}_{\mathrm{S}}=$ beam width +septum thickness + safety margin $=11.2(99 \%)+10+4=34.4 \mathrm{~mm}$, which requires $\theta_{\mathrm{k}}=-2.00 \mathrm{mrad}$ and consequently $\Delta \mathrm{x}_{\mathrm{s}} \mathrm{s}=-2.17$ mrad. The negative value of $\Delta x$ 's due to a relatively large and positive value of $\alpha_{S}=1.87$ is not ideal since it requires an extra kick from the septum to compensate it. It should also be noted that a longer bunch length $(\sim 110 \mathrm{~ns})$ will cause some horizontal emittance blow-up as the FKG10 strength varies $\pm \sim 5 \%$ within the bunch. 


\section{II.C. Instrumentation}

There are essentially minimum beam instruments that are specific to FEB in the AGS:

- two pairs of standard AGS short local loss monitors both at the upstream and at the downstream end of ssG10; one is connected to the G10 beam inhibit system for FKG10 protection, and another is to monitor possible beam loss through analog signals.

- one pair of short local beam loss monitors at ssH10us and ds.

- a standard AGS flag (1 mm thick) at the upstream end of SMH10 to monitor the position and profile of the bunch that is kicked by FKG10.

The extracted beam profile can be measured by digitizing images on the first flag at the beginning of the Uline (uf1). The $\mathrm{U}$ and $\mathrm{V}$ lines are intensively equipped with various types of instruments (loss monitors, position monitors, flags, current transformers etc.) to measure the extracted beam parameters and losses.

The new CYTEC Mux system for monitoring analog signals from FEB extraction devices (discharge currents and capacitor bank voltages for BLWs, FKG10, SMH10) has been fully implemented and operational throughout this commissioning and run period with protons.

\section{COMMISSIONING and RUN With PROTON BEAM}

On 3 May the FEB/V(p) commissioning with low intensity protons was successfully performed following the AGS-OPM-TPL 96-03, extracting 0.3-0.5 $\times 10^{12}$ protons in one bunch at $24 \mathrm{GeV} / \mathrm{c}$ to the $\mathrm{V}$ dump located at the end of the newly constructed $\mathrm{V}$ line. The extracted beam was transported to the 4 degree bend in the $U$ line to the bending dipoles vd3-4 where it was deflected about 3 degrees westward to the $V$ line and into the $\mathrm{V}$ target area. The FEB run continued until 3 July using the context switch on-request mode for the g-2 commissioning efforts lead by G. Bunce and H. Brown(EP\&S/E821), which included: 1) V line, 2) V-target tests at various bunch intensities for $3.1 \mathrm{GeV} / \mathrm{c} \pi / \mathrm{p}$ production 3)secondary $\mathrm{V} 1$ line, 4) the storage ring injection.

The H10 flag located the upstream end of SMH10, one of the few diagnostic tools available for FEB, was unfortunately stuck at the half way point and then broken mechanically at the end of May and was not available for the rest of the run. The CCD camera for uf1 (fist flag in U line) was radiation damaged by the HEP/SEB operation and was replaced with a standard video camera.

\section{IIII.A. Machine Setup for FEB}

The following FEB files created before the commissioning were loaded on AGS User\#2:

AgsMainMagnet, Ags/TuneControl, Ags/ChromControl, AgsRF/RadSteer, AgsRF/Voltage and AgsExtractQ-skew.

The beam with 8 bunches was accelerated to the AGS FEB flattop $(24.0 \mathrm{GeV} / \mathrm{c})$ at a low intensity of 2$3 \cdot 10^{12}$ ppp to setup the FEB bumps and to measure the orbit deformations by AgsOrbitDisplay which required at least $2 \cdot 10^{12} \mathrm{ppp}$ with multiple bunches. The analog outputs of the discharge current waveform from the four FEB backleg windings are shown in Figure 5. Figure 6 shows measured FEB extraction bumps by AgsOrbitDisplay with a prediction from MAD using the actual setpoint values at the FEB working point $\{\mathrm{Qh}, \mathrm{Qv}\}=\{8.74,8.77\}$. The raw PUE data are multiplied by a scale factor of 3.83 to match with the MAD prediction as a full PUE calibration has not been done. As you can see, there is a good agreement between the measured and predicted orbits in an overall shape, including the residual perturbations outside the bumps.

The $\mathrm{f}_{\mathrm{rf}}$ (beam revolution frequency $\mathrm{x} 8$ ) on the flattop was measured to check the beam momentum $\mathrm{p}$ and the mean radius $<\mathrm{dR}>$ as shown in Figure 7. Using the known machine parameters $(\rho, R, y)$ and the FEB setup parameters ( $B=0.93775 \mathrm{~T}$, and $\mathrm{h}=8$ ), we can calculate $\mathrm{f}_{\mathrm{rf}}$ to be 2969316 (257) $\mathrm{Hz}$ (on bumps) for $\mathrm{p}=24.0$ $\mathrm{GeV} / \mathrm{c}$ and $\langle\mathrm{dR}\rangle=\sim 0.0 \mathrm{~mm}$ with $\mathrm{dR} / \mathrm{df} \mathrm{rf}=-0.0487 \mathrm{~mm} / \mathrm{Hz}$. The measured value of $\mathrm{f}_{\mathrm{rf}}$ was $2969312(265)$ $\pm 15 \mathrm{~Hz}$ at RadSteerCommand $=5.6 \mathrm{~V}$, consisting with the desired values within an accuracy of $\mathrm{dp} / \mathrm{p} \leq \pm 0.1$ $\%$ and $<\mathrm{dR}>\leq \pm 1.5 \mathrm{~mm}$. 


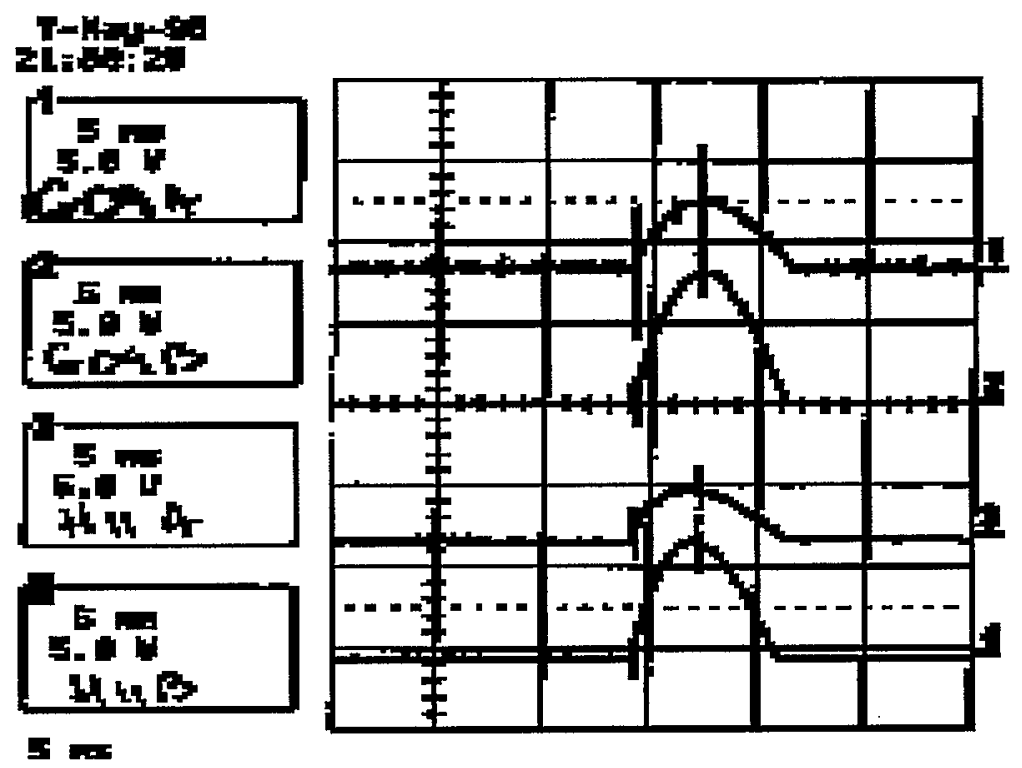

Figure 5: BLW's discharge current waveforms. ; 1) G09A_I, 2) G09B_I, 3) H11A_I and 4) H11B_I.

FEB bumps at $p=24.0 \mathrm{GeV} / \mathrm{c}$ for $V$-line

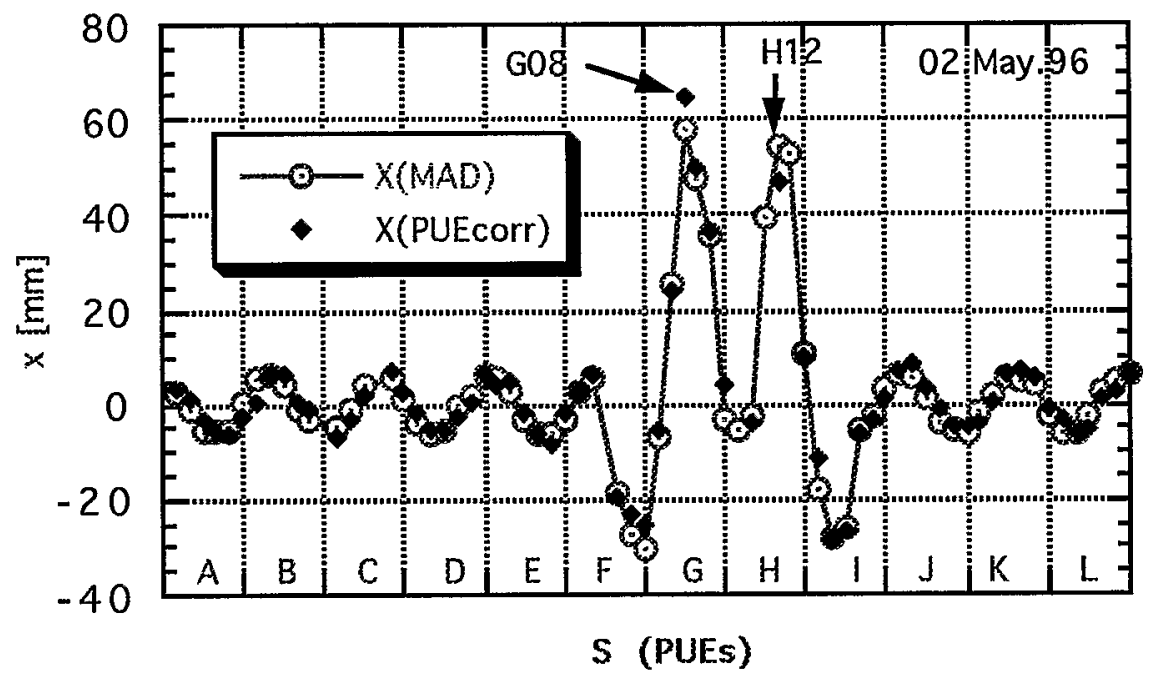

Figure 6: AgsOrbitDisplay Output of FEB Bumps: BLWG09 $=\{\mathrm{Ia}, \mathrm{Ib}\}=\{437,856\}$ A and $\mathrm{BLWH11}=\{\mathrm{Ia}, \mathrm{Ib}\}=\{434,818\} \mathrm{A}, \mathrm{x}(\mathrm{PUEcorr})=\mathrm{x}(\mathrm{PUEraw}) * 3.83$. 


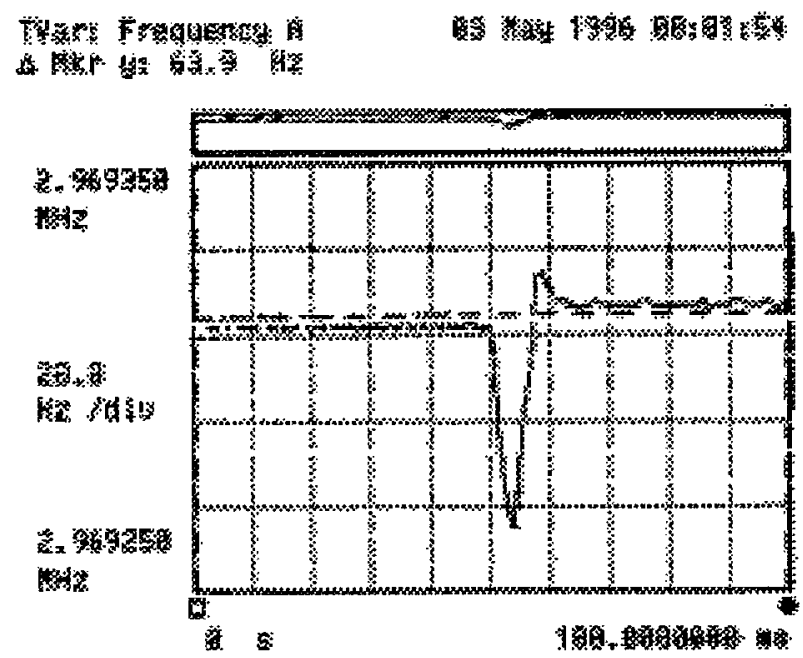

Figure 7: $A f_{\text {rf }}$ measurement on the flattop with the FEB bumps. Ver.: $20 \mathrm{~Hz} / \mathrm{Div}$ and Hor.: $10 \mathrm{~ms} / \mathrm{Div}$.

\section{III.B. FEB/SBE Extraction}

We switched to one Booster transfer having just one bunch of $\sim 1 \cdot 10^{12}$ protons and accelerated it in the AGS to avoid unnecessary beam loss in AGS. After turning on FKG10 and SMH10 at initial setpoint values, $\mathrm{I}_{\mathrm{k}}=$ $1.6 \mathrm{kA}$ and $\mathrm{I}_{\mathrm{S}}=16.7 \mathrm{kA}$, the beam spot was observed on the H10 flag, uf 2 and vf 326 (V target flag). The beam full width was $\pm \sim 9.1 \mathrm{~mm}$ horizontally and $\pm \sim 6 \mathrm{~mm}$ vertically on the $\mathrm{H} 10$ flag and was very flat vertically on vf325 for a beam of $\sim 0.4 \cdot 10^{12} \mathrm{ppb}$ (see Fig. 10). AgsLossMonitor indicated that there were no beam loss at FKG10 and a small loss at SMH10 due to the H10 flag insertion. Figure 8 shows current waveforms of SMH10 with BLWG09B and FKG10 with a bunch.
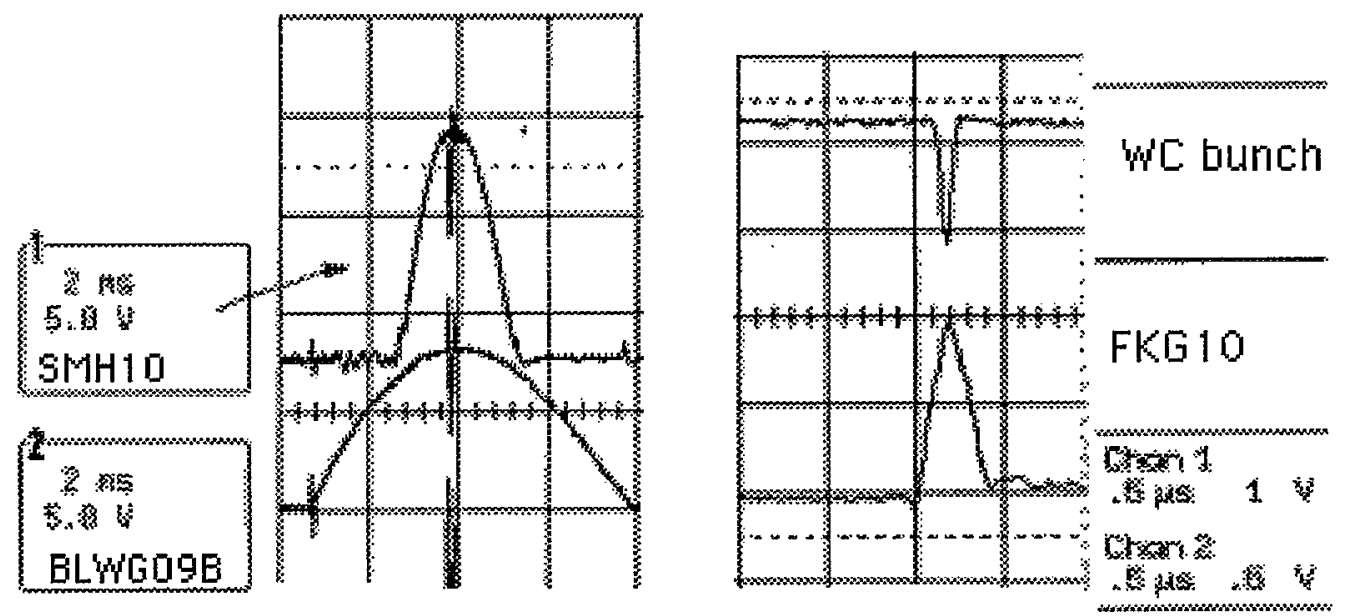

Figure 8: Left) SMH10 current waveform with one of BLWs and Right) FKG10 current waveform with a wall current monitor output of a bunch.

\rceil It was found later by $\mathrm{H}$. Brown that one of the $\mathrm{U}$ line quads, uq6, had a wrong polarity. 
The FEB/SBE(p) run with a low intensity beam $\left(0.2-0.5 \cdot 10^{12} \mathrm{ppb}\right.$ ) continued for the $\mathrm{V}$ line instrumentation test and various fault studies until 15 May. During this period:

- a standard video of uf1 became available.

- the H10 flag stuck at the middle position.

- SMH10 with a resonant charging module became unreliable, varying the discharge current by a few percent pulse-to-pulse and causing beam position variations on uf1. We disconnected the resonant charging module from the SHH10 power supply on 10 May and the SH10 reference current $\mathrm{I}_{\mathrm{S}}$ had to be increased from 16.7 A to $19.7 \mathrm{~A}$ to center the beam spot on uf1. It is likely that the SMH10 calibration value might have changed. -the polarity of uq6 was reversed and the expected round beam spot was observed on vf 325 as shown Figure 9.

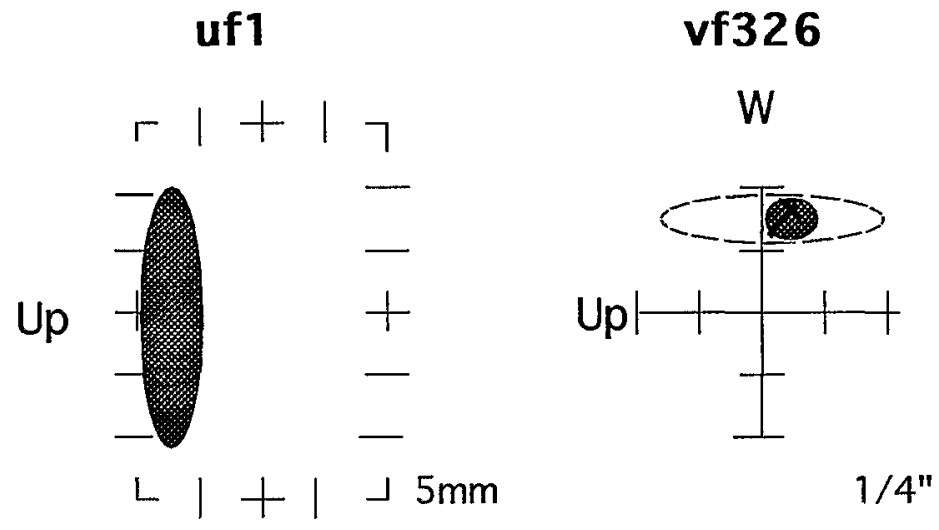

Figure 9: Beam spots observed on uf1 and vf326 (0200 on 08 May 96).

\section{III.C Run for the V-target Test at Various Intensities}

The $\mathrm{V}$ target for $3.1 \mathrm{GeV} / \mathrm{c} \pi / \mu$ production must withstand high peak/average beam heating up to the full intensity of $6 \cdot 10^{13} \mathrm{ppp}$ or $7.5 \cdot 10^{12} \mathrm{ppb}$ for $\mathrm{h}=8$. The target rotates and is water-cooled. The first $\mathrm{V}$ target test was performed at $\sim 1.0 \cdot 10^{12} \mathrm{ppb}$ and the second one at $\sim 2.5 \cdot 10^{12} \mathrm{ppb}$ for 30 minutes each by focusing the beam on the target with $\mathrm{v}$ line quads, vq9-vq12. As the bunch intensity increased over $1.5 \cdot 10^{12} \mathrm{ppb}$, we observed that the beam losses at ssH10-H13 grew substantially. When we tried to increase the beam intensity beyond $\sim 2.5 \cdot 10^{12} \mathrm{ppb}$ on flattop, we had frequently G10 Beam Inhibit and observed some beam loss at ssG10. The machine also became unstable, failing AGS acceleration more often. However, by the end of the run a fair FEB/SBE performance had been obtained with a high intensity Booster-AGS beam setup (identical to the SEB setup up to debunching), which was able to extract the single bunch beam exceeding $5 \times 10^{12} \mathrm{ppb}$ per cycle for the final 30-minute high intensity $V$ target test. Typical $A B$ (Booster)-AGS intensity performance during the final $\mathrm{V}$ target test was:

$$
\begin{array}{ll}
\mathrm{AB} \text { _input }=36.5 \cdot 10^{12} \text { protons } & \text { AGS_cbm }=6.86 \cdot 10^{12} \mathrm{ppb} \\
\mathrm{AB} \text { _early }=27.5 \cdot 10^{12} \mathrm{p} / 2 \mathrm{~b} & \text { AGS_before } \gamma_{\mathrm{t}}=5.66 \cdot 10^{12} \mathrm{ppb} \\
\mathrm{AB} \text { _late }=20.5 \cdot 10^{12} \mathrm{p} / 2 \mathrm{~b} & \text { AGS_flattop }=5.22 \cdot 10^{12} \mathrm{ppb}
\end{array}
$$

On the other hand, partially due to larger transverse beam emittance ( $\geq 80 \pi \mathrm{mm}$-mrad), wider momentum spread and longer bunch length $(\sim 100 \mathrm{~ns})$ for the high intensity bunch beam and also probable SMH10 misalignment there were substantial beam losses at SMH10ds (see Fig. 10) and its downstream ssH11-H13. To minimize these losses to a few percent level FKG10 had to kick the beam at the near maximum strength $(\sim 2.0 \mathrm{kA})$ and the BLWG09 had to push the beam further into the FKG10 aperture. Increasing the SMH10 kick could have reduced the beam loss at SH10ds but had increased the losses at ssH11-H13. 


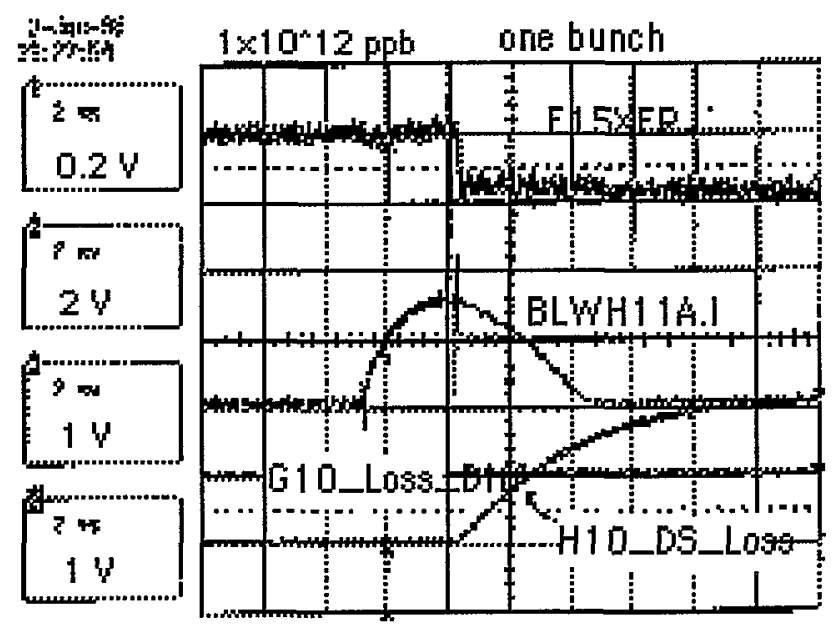

Figure 10: 1) F15 XFR, 2)BLWH11A_I, 3)G10_loss_Diff and 4)H10_DS_loss

\section{III.D Double Single-Bunch Extraction}

Tests of double SBEs per AGS cycle had been carried out with two-bunch acceleration in the AGS. All FEB extraction devices had double-pulsed at $100 \mathrm{~ms}$ part at equal strength and the first SBE was successfully performed but not the second one as shown in Figure 11. After the first SBE, there were big beam losses at ssH10ds as well as ssG10 that triggered the G10 Beam Inhibit system to turn off the rf. It was found that the radial loop system could not keep the same mean radius for the second bunch left in the ring after the first SBE was performed. The beam became unstable, drifted away radially outward and crashed to the FKG10 and SMH10 before the second SBE attempt as seen in Figure 12. The rf frequency loop was also tried but not successfully.

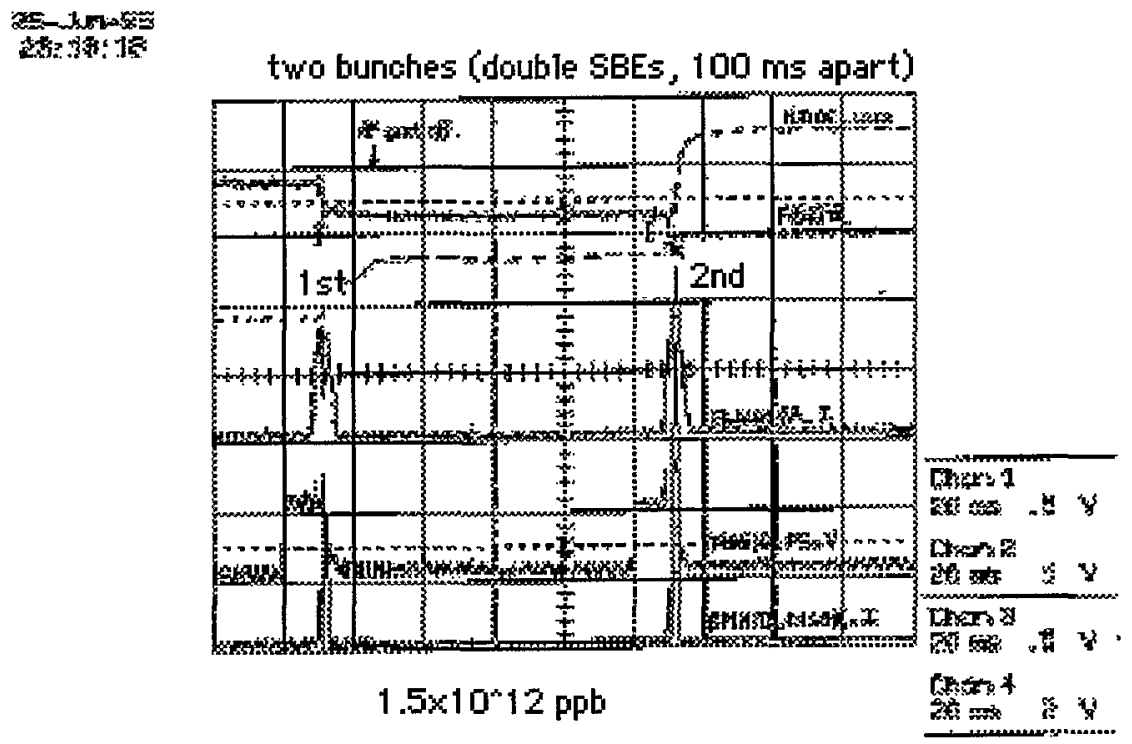

Figure 11: 1) F15XFR, 2) H10_DS_Loss, 3)BLWG09A_I, 4)FKG10_V,5) SMH10.Discharge.I 
$5-1+5$

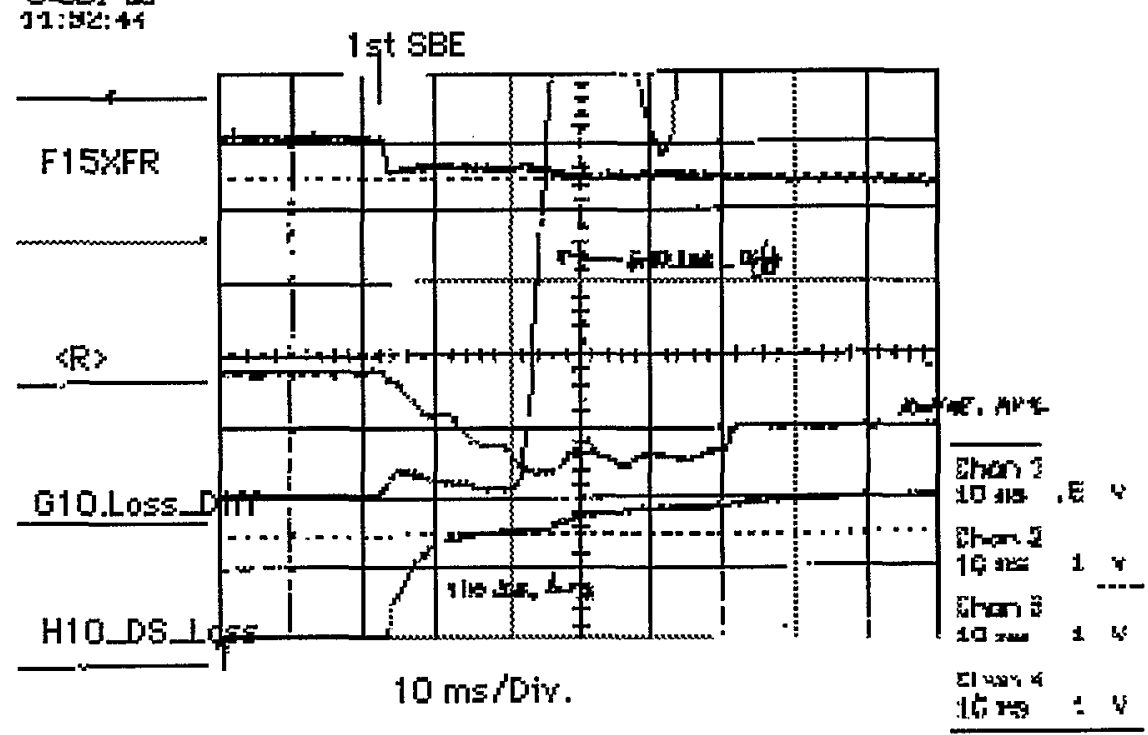

Figure 12: 1)FX15XFR, 2)Radial_Average, 3)G10_Loss_Diff and 4) H10_DS_Loss.

\section{III.D Miscellaneous}

-When the FY96 HEP/SEB(p) run had started at an intensity of $\sim 1.7 \cdot 10^{13}$ ppp and the local G10 loss monitor (G10_Loss_Diff) showed a small but distinct sharp peak for each Booster transfer during the AGS injection, the G10 injection bump was turned on at $\mathrm{I}=4 \mathrm{~A}$, which produced a bump of $-5 \mathrm{~mm}$ around ssG10 and eliminated the loss. As the intensity became higher the loss reappeared and triggered the G10 Beam Inhibit so that we had to increase the current to the maximum of $6 \mathrm{~A}$ to minimize the loss. If the G10 injection bump was off, the beam would loose $5-20 \%$ of its intensity depending on the exact machine conditions at AGS injection. Note that since the G10 kicker is made of a brittle ceramic-type material and its downstream end is the most outside limiting aperture, it must be protected from possible beam scraping.

- The secondary beam line V2 was also commissioned and the $\pi / \mu$ beam was transported to the $g-2 \mu$ storage ring on 11 June 1997 by G. Bunce and H. Brown.

-AgsLossMonitor(X-version) became functional with multiple time windows and CT readings. It was an invaluable diagnostic tool especially since we lost the H10 and ufl flags. Note that AgsIpm was not available and AgsOrbitDisplay did not work for one bunch beam.

- During the HEP/SEB cycles there was substantial injection loss around ssH12-H13 due to vertical beam scraping. Before FEB/V(p) commissioning, HP surveyed around the FEB extraction area and measured $600 / 700 \mathrm{mrem} / \mathrm{hr}$ at ssH13us/ds. This loss should be avoided or be moved somewhere else.

-The intensity dependency on the bunch length and beam size was studied by increasing the Chopper width. The beam size (FWHM) observed on uf 2 was varied from $10.3 \mathrm{~mm}$ for $1.4 \cdot 10^{12} \mathrm{ppb}$ at $125 \mu \mathrm{s}, 18.6 \mathrm{~mm}$ for $2.710^{12}(175 \mu \mathrm{s})$ and $17.1 \mathrm{~mm}$ for 3.3.10 $12(325 \mu \mathrm{s})$. However, the bunch length remained constant at $l_{\text {bunch }}($ full $)=\sim 90 \mathrm{~ns}$.

-Table 4 summarizes the machine conditions, FEB parameters, and performance for the FEB/V(p) run for the $\mathrm{V}$ and $\mathrm{V}$ target commissioning. 
Table 4: A Summary for FY96 FEB/V(p) Commissioning and Run

\begin{tabular}{|c|c|c|}
\hline User & $\begin{array}{l}\text { g-2(E821-L. Robert/W. } \\
\text { Morse) }\end{array}$ & G. Bunce, H. Brown \\
\hline Comm.Date/Time & $\begin{array}{l}\text { 02.May.96[Th]: } \\
1600-0800\end{array}$ & $\begin{array}{l}\text { beam on V-target flag } \\
\text { (vf325) }\end{array}$ \\
\hline Phase I & SBE to U $>V->V$ Dump & $\sim 1 \cdot 10^{12} \mathrm{ppb}$ \\
\hline Phase II & to V Target & $1,2.5,5 \cdot 10^{12} \mathrm{ppb}$ \\
\hline Phase III & to $V 1->\mu$-storage ring & $1-3 \cdot 10^{12} \mathrm{ppb}$ \\
\hline Run Period & 03.May.96-03.July.96 & \\
\hline Run Mode & $\begin{array}{l}\text { Context Switching with } \\
\text { SEB }\end{array}$ & $\begin{array}{l}\text { on-request mode and } \\
330 \text { min. dedicated runs }\end{array}$ \\
\hline Ions $[A, Z]$ & protons & \\
\hline Momentum[p] & $24.0 \mathrm{GeV} / \mathrm{c}$ & \\
\hline Intensity & $1->510^{12} \mathrm{ppb}$ & \\
\hline FlattopLength & $1100-(2900) \mathrm{ms}$ & \\
\hline FlattopField & $0.93775 \mathrm{~T}$ & $\mathrm{p}=24.0 \mathrm{GeV} / \mathrm{c}$ \\
\hline $\mathbf{f}_{\mathrm{rf}}$ & $2969312(265) \mathrm{Hz}$ & (on bumps) at $<\mathrm{dR}>=0$. \\
\hline FEB TimeWindow & $1200-1600 \mathrm{~ms}$ & from To \\
\hline AGS Rep.Rate & $(3.6 \mathrm{~s})$ & same as HEP/SEB \\
\hline Harmonic & $\mathrm{h}=8$ & \\
\hline $\mathrm{e}^{*} \mathrm{~T}(95 \%)$ & $\sim 75-90 \pi \mathrm{mm}-\mathrm{mrad} ?$ & \\
\hline $\mathrm{e}^{*} \mathrm{~L}(95 \%)$ & $\sim 5 \mathrm{eV}-\mathrm{sec} ?$ & \\
\hline lbunch(full) & $90-110 \mathrm{~ns}$ & \\
\hline Nbunch & $1(2)$ & in $\mathrm{AGS}$ (one $\mathrm{AB}$ transfer) \\
\hline WorkingPoint & $\{\mathrm{Qh}, \mathrm{Qv}\}=\{8.73,8.77\}$ & on FEB bumps \\
\hline Highfield Sexts & $\{\mathrm{ISh}, \mathrm{ISv}\}=\{50,0\} \mathrm{A}$ & \\
\hline BLWG09 (bump) & $\{\mathrm{Ia}, \mathrm{Ib}\}=\{448,872\} \mathrm{A}$ & \\
\hline BLWH11 (bump & $\{\mathrm{Ia}, \mathrm{Ib}\}=\{423,808\} \mathrm{A}$ & \\
\hline FKG10 (kicker) & $\mathrm{I}=\sim 1.95 \mathrm{kA}$ & \\
\hline SMH10 (septum) & $\mathrm{I}=\sim 19.7 \mathrm{kA}$ & \\
\hline IBG10 (Inj.bump) & $3000 \mathrm{CNT}$ (max) & with 6 A PS \\
\hline FlagH10 & @ssH10us & broken \\
\hline AGS SLMs & $\begin{array}{l}@ \text { @sGG10us\&ds and } \\
\text { ssH10us\&ds short LMs }\end{array}$ & \\
\hline Tools & $\begin{array}{l}\text { CT,PUE,(IPM),WC, } \\
\text { AGSLM,uf1,vf } 325\end{array}$ & \\
\hline
\end{tabular}




\section{CONCLUSIONS AND OUTLOOK}

- The FEB extraction system was commissioned with proton beam, extracting one bunch per AGS cycle to the U-line at $24 \mathrm{GeV} / \mathrm{c}$ and run for the $\mathrm{V}$ line and $\mathrm{V}$ target commissioning efforts lead by $\mathrm{G}$. Bunce and $\mathrm{H}$. Brown using the context switch on-request mode.

- There were some beam losses at ssH10-H13 during extraction for $>310^{12} \mathrm{ppb}$.

- The maximum extracted beam intensity was $\sim 5.210^{12} \mathrm{ppb}$.

- For the next FEB run for the g-2 experiment, we hope that we can have an adequate machine time for fine tuning of FEB and:

- to test the trim backleg windings for better FEB bumps with AgsOrbitDisplay and obtain good calibrations,

- to perform multiple single-bunch extraction (2 to 4 (8?) SBEs/cycle)

- at $30 \mathrm{~Hz}$,

- up to $7.5 \cdot 10^{12} \mathrm{ppb}$,

- with $\sim 100 \%$ extraction efficiency,

- to measure fully the internal beam parameters $\left(\varepsilon \mathrm{T}, \varepsilon_{\mathrm{L}}, \sigma_{\mathrm{p}} / \mathrm{p}\right)$ and pulse-to-pulse, day-by-day beam stability.

\section{ACKNOWLEDGMENTS}

The FEB/V commissioning with proton beam was performed collectively by the Accelerator and EP\&S staff in the AGS Department. We thank specially the RF group led by M. Brennan to setup the AGS complex for just one bunch acceleration in both Booster and AGS. We also thank B. Tamminga and K. Brown for their extra help on FEB and T. Satogata(RHIC) for his help on U line beam instrumentation and J. Geller for V line instrumentation.

We would like to thank many members of the AGS Department who have helped to construct the FEB system and make this commissioning successful: A. Dunbar (bumps), J. Tuozzolo (kicker), E. Rodger (ejector), W. Zhang (kicker PS), R. Lockey (ejector PS), E. Gill (injection bump), E. Keith-Monnia (controls-software) N. Schumburg(controls-hardware), J. Skelly (controls-datacon), P. Ingrassisa and MCR staff, and many others.

\section{REFERENCES}

[*] AGS FEB has a www home page: http://www.ags.bnl.gov/ tanaka/agsfeb.html.

[1] AGS Experiment 821, “A New Precision Measurement of the Muon g-2 Value at the Level of 0.35 ppm" and the Design Report.

[2] M. Tanaka, "FEB/SBE(Au): Commissioning and Run for the FY1996 AtR TL Commissioning", AGS/AD/Tech Note No. 453(1996).

[3] M. Tanaka, "The New Fast Extraction System at the AGS", AGS/AD/Tech Note No. 347 (1991).

[4] M. Tanaka et. al., "The BNL AGS Accelerator Complex with the new Fast Extraction System", 1995, IEEE 95CH35843, Vol.3, 1930-1932 (1996). 